\title{
Cue-Induced Brain Activity Changes and Relapse in Cocaine-Dependent Patients
}

\author{
Thomas R Kosten*,', Barbara Ellen Scanley², Karen A Tucker', Alison Oliveto', ${ }^{1,3}$ Chekema Prince ${ }^{4}$, \\ Rajita Sinha ${ }^{2}$, Marc N Potenza ${ }^{2}$, Pawel Skudlarski ${ }^{5}$ and Bruce E Wexler ${ }^{2}$ \\ 'Department of Psychiatry, Yale University School of Medicine, VA Connecticut Healthcare System, West Haven, CT, USA; ${ }^{2}$ Department of \\ Psychiatry, Yale University School of Medicine, Connecticut Mental Health Center, New Haven, CT, USA; ${ }^{3}$ Department of Psychiatry, University of \\ Arkansas for Medical Sciences, Little Rock, AR, USA; ${ }^{4}$ Department of Brain and Cognitive Sciences, Massachusetts Institute of Technology, \\ Cambridge, MA, USA; ${ }^{5}$ Department of Radiology, Yale University School of Medicine, New Haven, CT, USA
}

\begin{abstract}
This study used functional magnetic resonance imaging ( $(\mathrm{M} R \mathrm{RI})$ to examine the association between brain activation during exposure to cocaine-related cues and relapse to drug use in cocaine-dependent (CD) patients. We imaged 17 CD subjects during a 2-week in-patient stay. The subjects then entered a I0-week outpatient placebo-controlled, double-blind randomized clinical trial where urine toxicologies were assessed three times weekly to calculate the treatment effectiveness score (TES). Worse TES correlated with BOLD activation in the left precentral, superior temporal, and posterior cingulate cortices (PCC), and right middle temporal and lingual cortices $(R>0.65$; $P<0.005$ ). The left PCC activation also distinguished eight nonrelapsers (TES above mean and completed treatment) from nine relapsers. Cocaine-free urines were significantly greater in the nonrelapsers (92\%) than in the relapsers (66\%), who also remained in treatment for an average of only 3.2 weeks. Self-reports of craving during fMRI did not differ between nonrelapsers and relapsers and did not correlate with TES. Relapse to cocaine abuse was associated with increased activation in the sensory association cortex, the motor cortex, and PCC while viewing images of cocaine-related cues. These results suggest that relapse to cocaine abuse is associated with increased brain activation to cocaine cues in sensory, motor, and cognitive-emotional processing areas. This physiological activation was a better predictor of relapse than subjective reports of craving, and may be a useful target for treatment development.

Neuropsychopharmacology (2006) 31, 644-650. doi: I0.1038/sj.npp. I 30085 I; published online 10 August 2005
\end{abstract}

Keywords: cocaine; fMRl; posterior cingulate cortex; treatment outcome; drug cues; sertraline

\section{INTRODUCTION}

Exposure to cocaine-related cues activates a wide range of brain areas including frontal and temporal cortex regions and limbic structures such as the anterior and posterior cingulate (Childress et al, 1999; Garavan et al, 2000; Maas et al, 1998; Wexler et al, 2001; Wilson et al, 2004). The possible association of these brain activations with relapse to drug use has not been examined in cocaine-dependent (CD) patients. Relapse to drug use has not been well predicted by self-reported craving in response to drug related cues but responses that occur before craving is reported may have better predictive value (Tiffany and

\footnotetext{
*Correspondence: Dr TR Kosten, Department of Psychiatry, Yale University School of Medicine, I5ID - Bldg 35, VA Connecticut Healthcare System, 950 Campbell Avenue, West Haven, CT 065 I6, USA, Tel: 203932 57।I ext 7438, Fax: 203937 4915,

E-mail: thomas.kosten@yale.edu

Received 13 September 2004; revised 3 June 2005; accepted 7 June 2005

Online publication: 28 June 2005 at http://www.acnp.org/citations/ Npp062805040420/default.pdf
}

Carter, 1998). Examples of responses that can occur before craving is reported include neurophysiological arousal to alcohol cue exposure. In a recent study abstinent alcoholics, who reported little subjective response to alcohol-related cues, had a strong inhibition of startle responses, which reflect relatively automatic rather than cognitive processes (Mucha et al, 2000). This type of automatic response below conscious awareness might reflect an important drive for using cocaine and be detected using functional brain imaging with BOLD-MRI during the initial $30 \mathrm{~s}$ of exposure to videotape cocaine cues. In our previous use of the videotapes that are used in the current study (Wexler et al, 2001), strong and early (within 30 s) activation of sensory and limbic areas were evident prior to report of subjective craving responses. Moreover, activation seen only in $C D$ subjects during the initial period of viewing the cocaine cue tapes was present in both subjects who did and those who did not go on to report experiences of craving. These responses below awareness may be more strongly associated with return to drug use than reported craving or any brain activations correlated with craving. Currently, challenges exist in predicting how well an individual seeking treatment 
for cocaine dependence will fare during treatment. There would be significant clinical implications if treaters could use a priori information (that is, data available prior to treatment) to influence treatment decisions. Brain imaging techniques hold the potential to examine whether any specific patterns of brain activation can predict treatment outcome and more specifically, relapse to drug use. As an example of this promise, recent studies of depressive disorders have suggested that the extent of anterior cingulate activation before starting antidepressants can predict depressive symptom resolution (Davidson et al, 2003; Mayberg et al, 1997).

In the current pilot study, we examined the potential of brain activity changes in response to cocaine cues to predict relapse to cocaine use as assessed using the Treatment Effectiveness Score (TES) (Ling et al, 1997). The TES is the sum of all cocaine-free urines during the treatment trial, and we correlated the TES with regions of brain activation on fMRI during cocaine cue exposure. For a subsequent analysis, all the $\mathrm{CD}$ patients who remained in treatment and had a TES above the mean, which indicated relatively little cocaine use, were classified as nonrelapsers and the remaining $\mathrm{CD}$ patients were defined as relapsers.

We hypothesized that, during the first $30 \mathrm{~s}$ of viewing of cocaine cues by treatment-seeking CD subjects, activation of brain regions previously implicated in cocaine craving, including areas of frontal and anterior and posterior cingulate cortices, would be inversely associated with TES scores and related to relapse. Specifically, we predicted that individuals with TES scores below the mean TES score would show more activation in these brain regions.

\section{PATIENTS AND METHODS}

\section{Patients from Clinical Trial}

A total of, $33 \mathrm{CD}$ patients participated in a 12-week, randomized, placebo-controlled, double-blind clinical trial examining the efficacy of the SSRI sertraline at $200 \mathrm{mg}$ daily in depressed CD patients. Patients were admitted to the VA CT Healthcare System Residential Q-house for 2 weeks in order to achieve initial cocaine abstinence and be inducted onto the study medication. The fMRI was performed prior to sertraline induction. After the fMRI session, sertraline induction was begun and subjects received the maintenance dose of sertraline by the beginning of week 4 . After 2 weeks, patients were discharged from the residential unit and continued to participate on an outpatient basis for 10 weeks, during which time they received weekly individual cognitive behavioral therapy (CBT) and submitted urines thrice weekly for urine toxicologies.

The fMRI session was conducted in 21 of the 33 patients who were medication and drug-free (median 3 days post admission and 10 days since last reported cocaine use). Nine patients declined the fMRI and three declined it right before it was scheduled. The fMRI participants were comparable demographically and on baseline drug use to the fMRI nonparticipants. Four of 21 patients were excluded due to movement during the fMRI. The remaining 17 were right-handed (12 male; 13 African American and four Caucasian; mean age $=37$ years, $S D=4)$. The patients all met the DSM-IV criteria for cocaine dependence on the
SCID and used cocaine for an average of 6 years with 20 days of use in the month before entering this study (First et al, 1995). No psychotic, other current substance dependence or major psychiatric Axis I disorders were found on the SCID, except nicotine dependence in all patients. Depressive symptoms were assessed using the Hamilton Depression Scale (Hamilton, 1960). No subjects took psychotropics the month before the study, had a history of neurological illness or injury, or had abnormalities on structural MRI. All gave written informed consent on a Yale approved protocol.

\section{Stimulus Videotapes}

Two different videotapes of an actor pretending to smoke cocaine and get a rush were presented in the same order to all subjects. These 3-min tapes were preceded by $60-\mathrm{s}$ baseline periods from C-SPAN describing vegetable prices. The neutral baseline video segments were used to control for visual and auditory stimulation aroused by viewing images of individuals speaking. Immediately after viewing each tape through video goggles, the subjects were asked to rate from 0 to 10 the peak craving intensities. In our previous study these tapes reliably elicited craving (Wexler et al, 2001). We compared the fMRI response during the initial 1-min baseline to the response during the cocaine cue tape, with a focus on the first $30 \mathrm{~s}$ of viewing the cocaine cue tape.

\section{Functional MRI and Statistical Analyses}

We used a 1.5-T GE Signa MRI system with resonant gradients for echoplanar imaging and T1-weighted spin echo sagittal anatomical images $(\mathrm{TE}=14 \mathrm{~ms}, \mathrm{TR}=500 \mathrm{~ms}$, field of view $=24 \mathrm{~cm}$, slice thickness $=5 \mathrm{~mm}, \quad$ gap $=0$, $192 \times 192$ data matrix) for slice localization. Next, 14 T1weighted oblique axial slices $(\mathrm{TE}=14 \mathrm{~ms}, \mathrm{TR}=500 \mathrm{~ms}$, field of view $=20 \times 20 \mathrm{~cm}$, slice thickness $=8 \mathrm{~mm}$, skip $=1 \mathrm{~mm}$, $192 \times 192$ data matrix) were acquired parallel to the plane transecting the anterior and posterior commissures, covering the brain from the inferior temporal sulcus to the most superior portion of the cortex. Functional images were obtained using a single-shot echoplanar gradient-echo sequence (flip angle $=60^{\circ}, \mathrm{TE}=60 \mathrm{~ms}, \mathrm{TR}=1.5 \mathrm{~s}$, field of view $=20 \times 20 \mathrm{~cm}, 64 \times 64$ data matrix, slice thickness $=8 \mathrm{~mm}$, skip $=1 \mathrm{~mm})$. Head movement was evaluated by measuring changes in the center of mass of the functional images over time and corrected for three translational directions and possible rotations (Friston et al, 1995). Four of 21 patients were removed from analyses because their head motion exceeded $0.75 \mathrm{~mm}$ from the beginning to the end of a particular tape. The corrected images were spatially filtered using a Gaussian filter with a full width at half maximum of $6.25 \mathrm{~mm}$.

For analyses, each subject first had the average echoplanar imaging signal during the baseline compared to the average signal during the first $30 \mathrm{~s}$ of cue exposure in each voxel from all acquisitions. We also examined the full $3 \mathrm{~min}$ of cue exposure compared to the baseline. These average signal maps and the anatomic images from individual subjects were transformed into the standardized Talairach atlas coordinate system (Talairach and Tournoux, 1988) in 
order to combine data across subjects. Second, the average signal maps were used to compute standard linear contrast measures (Hays, 1988). Under the null hypothesis of no effect, the expected value of the mean of this contrast across subjects is equal to zero. We then used a Monte Carlo procedure to generate a distribution of task-related $t$-values in order to estimate the significance of the observed linear contrast at each voxel (Manly, 1997; Shaywitz et al, 1999; Skudlarski and Gore, 1999). This procedure creates the population distribution for each voxel by repeatedly calculating the value of the contrast when the $t$-values of one-half the subjects, randomly chosen, have a reversed sign. This randomization was performed 1000 times, generating a sampling distribution of the linear contrast measures. The observed linear contrast measure, calculated without sign reversal, was assigned a $P$-value on the basis of its position in this distribution. All reported $P$-values were derived from this procedure.

The subjects' fMRI responses were correlated with the TES and then used to compare nonrelapsers to relapsers (Ling et al, 1997). The TES score is the total number of cocaine-free urines provided by a subject during the outpatient portion of the study and ranged from 0 to 30 , the best outcome. This measure is superior to simple percentages of cocaine-positive urines in relapse prevention trials for two reasons. First, the TES does not give the same outcome weighting to a patient who leaves after 1 day as a drug-free outpatient, as to a patient who remains in the 10 weeks of outpatient care and remains drug-free. Second, it does not assume that missing urines after a patient drops out of treatment are cocaine positive. Thus, it is a relatively balanced outcome for considering both treatment retention and remaining drug-free.

Whole brain voxel based correlation analyses were conducted to examine the association between BOLD signal change and TES scores. We correlated the TES with the mean signal at each individual voxel, and displayed clusters of 30 contiguous voxels which each showed correlation coefficients greater than $0.64(P<0.005)$. The resulting activation map showed clusters that had lower TES (relapse to cocaine use and poor retention). Covariance adjustments for Hamilton depression scores were also carried out between the TES and brain activation. Relapsers were defined as those patients who remained in outpatient treatment for less than 10 weeks and had no or few cocainefree urines leading to a TES score less than the mean TES of 15 for all the patients. The remaining patients were defined as nonrelapsers. Significant differences in brain activation between treatment nonrelapse and relapse groups were evaluated by switching the group assignments in randomly chosen subsets of one-half of the subjects and applying the randomization procedure to periods of tape viewing with the significance level set at $P<0.01$.

\section{RESULTS}

The BOLD response to the first $30 \mathrm{~s}$ of tape viewing compared to the baseline response showed five clusters that had significant correlations with the TES $(r=0.64$; $P<0.005)$. Table 1 shows the size and $x, y$, and $z$ coordinates for the center of mass of these five clusters. The largest areas of activation were the right lingual and left posterior cingulate cortices. The right superior temporal, and right inferior occipital cortices also were associated with the TES. No negative correlations were found between the TES and brain activation. The percentage of cocaine-free urines was positively correlated with the number of weeks subjects remained in the study $(r=0.9, P<0.001)$, as shown in Figures 1 and 2. As expected from this high correlation between urine results and treatment retention, the TES also was highly correlated with the percentage of cocaine-free urines $(r=0.9)$, and the correlations between cocaine-free urines and areas of brain activation were identical to those using the TES. Furthermore, no significant correlations were found between the Hamilton depression or craving scores and brain activation at $P<0.005$. The mean Hamilton score was 23.4 (SD 6.7) at baseline and significantly declined to 7.1 (SD 6.4) $(t=7.6 ; P<0.0001)$. Hamilton scores were not significantly correlated with TES $(R=-0.3)$. Moreover, covariance adjustment for depressive symptoms did not change the significance or location of any associations between TES and brain activation. Finally, the BOLD response to viewing the full 3-min cocaine cue tape compared to the baseline response demonstrated significant correlations with the TES in only two of the five voxel clusters found for the initial $30 \mathrm{~s}$ : the left precentral and the right superior temporal cortices.

Table I Brain Regions Showing Significant Correlations $(P<0.005)$ between Cocaine Cue Induced Activation and Treatment Effectiveness Score $(n=17)$

Talairach coordinates

\begin{tabular}{|c|c|c|c|c|c|c|}
\hline \multirow[b]{2}{*}{ Regions of activation } & \multirow[b]{2}{*}{ BA } & \multirow[b]{2}{*}{ Side } & & \multirow[b]{2}{*}{ Cluster size } \\
\hline & & & $\mathbf{x}$ & y & $\mathbf{z}$ & \\
\hline Posterior cingulate & 30 & L & -4 & -60 & 11 & 2767 \\
\hline Lingual gyrus & 19 & $\mathrm{R}$ & 21 & -68 & -5 & 3064 \\
\hline Inferior occipital gyrus & 19 & $\mathrm{R}$ & 41 & -69 & -5 & 1309 \\
\hline
\end{tabular}

Brain regions and corresponding Brodmann's areas are indicated, as well as $(x, y, z)$ locations in Talairach space for the average center of mass for an activity change. Negative $y$-values indicate brain posterior to the anterior commissure. Volume of activation is tabulated under 'Cluster size' as cubic millimeters.

Note: $\mathrm{BA}=$ Brodmann's area. $\mathrm{L}=$ left, $\mathrm{R}=$ right; Based on reading of randomization maps, as shown in Figure I. 


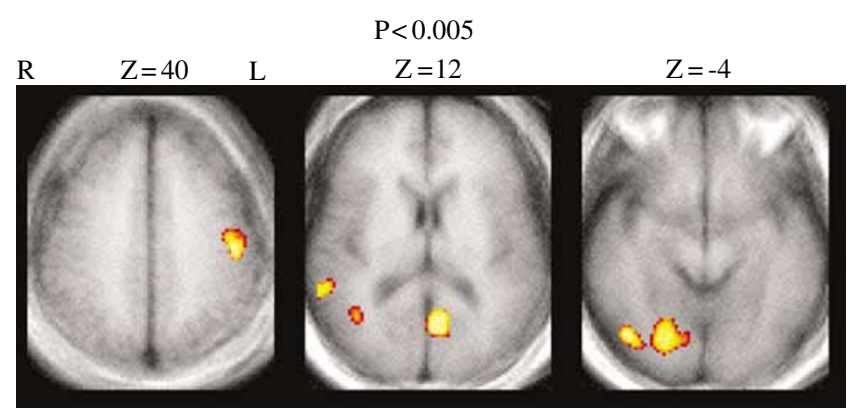

Figure I Displayed are brain regions showing significant correlations between cocaine cue-induced activation and Treatment Effectiveness Score (TES) for first $30 \mathrm{~s}$ of tape vs baseline. We show activations correlating at a significance threshold of $P<0.005(r=0.64)$, (yellow areas $P<0.00 I$ ). Cross-sectional slices are shown at $Z$ levels of 40, 12, and -4 . Left $L$ and right $R$ brain hemispheres are indicated above the pictures. The areas of activation are: (I) left precentral cortex (BA4) at $Z=40$, (2) left posterior cingulate (BA30) and right superior temporal (BA22) cortices at $Z=12$, (3) right lingual (BA|9) and inferior occipital (BA|9) cortices at $Z=-4$.

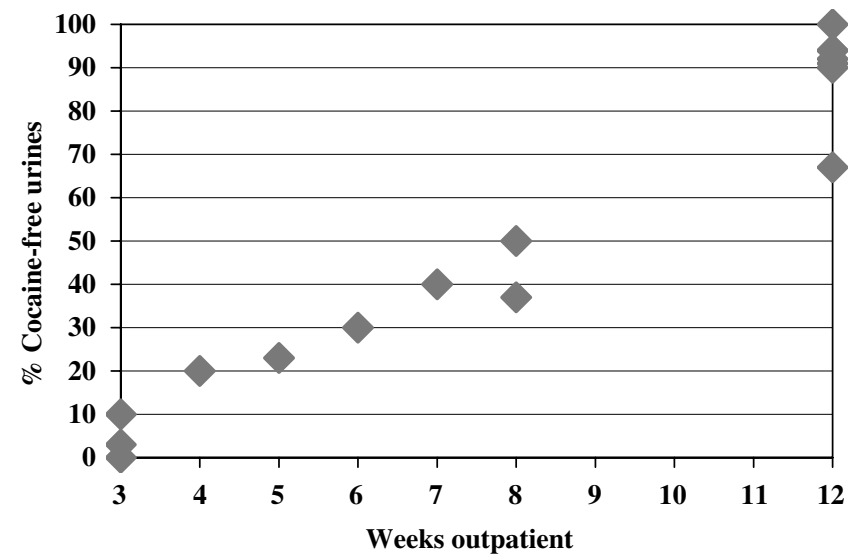

Figure 2 Regression line and individual subject values show the relationship between weeks in treatment and percentage of cocaine-free urines during the 12-week clinical trial. Three subjects who completed the 12 -week study had overlapping values in the percentage of cocaine-free urines. Overall correlation: $r=0.996, P<0.0001$.

Since the 17 subjects readily formed a cocaine abuse nonrelapse and relapse group based on treatment retention and urine toxicology as shown in Figure 2, we divided them and compared their fMRI responses. The 17 subjects included eight nonrelapsers, who all completed 2 weeks inpatient followed by the 10 -week outpatient trial and had a greater TES than the mean of 15 . The nine relapsers remained in outpatient treatment for 3.2 (SD 2.2, range 1-6 weeks) out of 10 weeks and had a significantly lower TES than nonrelapsers $(27$ vs 7$)(t=9.4 ; \mathrm{df}=15 ; P<0.001)$. Nonrelapsers also had more cocaine-free urines using the raw data (90 vs $66 \%)(T=2.4 ; \mathrm{df}=15 ; P<0.05)$ or imputing for missing data $(90$ vs $24 \%)(T=9.6 ; P<0.001)$. Five relapsers and six nonrelapsers were treated with sertraline and the rest with placebo $(P>0.4)$. Demographics, baseline drug use and craving did not differ between the two groups, as shown in Table 2 .

Based on our correlational findings with the TES and relatively few significant differences in brain activation
Table 2 Means (Standard Deviations) for Baseline Measures for Treatment Nonrelapsers vs Relapsers

\begin{tabular}{lcc}
\hline Measure & Nonrelapsers & Relapsers \\
$\mathbf{N}$ & $\mathbf{8}$ & $\mathbf{9}$ \\
\hline Age (years) & $39.5(9.0)$ & $39.2(5.8)$ \\
Sex (\#male/female) & $6 / 2$ & $6 / 3$ \\
Race (\#C/AA) & $1 / 7$ & $3 / 6$ \\
Education (years) & $13(1.0)$ & $12(1.4)$ \\
Employed (\%) & $75 \%$ & $55 \%$ \\
Cocaine use (\#days/mo) & $20(9)$ & $21(9)$ \\
Cocaine use (\#yrs) & $4.7(5)$ & $7.9(5)$ \\
Alcohol (\#days/mo) & $7(7)$ & $15(9)$ \\
Alcohol (\#yrs) & & $23(4)$ \\
Tobacco (pks/day) & $20(6)$ & $0.8(0.8)$ \\
Hamilton depression & $0.6(0.5)$ & $25(4)$ \\
Craving & $19(9)$ & $2.9(2.7)$ \\
\hline
\end{tabular}

aRace: C-Caucasian, AA-African American.

${ }^{b}$ Alcohol (\#days/mo): number of days using alcohol in month before entering study.

'Alcohol (\#yrs): years meeting abuse criteria for alcohol (nonrelapsers $n=4$; relapsers $n=6$ ).

between the cocaine cue and baseline conditions for the total sample, we compared the BOLD response between the nonrelapsers and relapsers during the initial $30 \mathrm{~s}$ minus the baseline. At a threshold of $P<0.01$, only two brain areas showed activation differences between cue and baseline conditions for the full sample of 17: the right temporal $(X$ 57, $Y-37, Z-4)$ and left occipital $(X-15, Y-35, Z-12)$. However, activation differences between cue and baseline conditions for the contrast of relapsers to nonrelapsers showed significant activation differences in two different clusters that corresponded well to the correlations between the TES and brain activation. Figure 3 , shows that the cluster in the right precentral cortex is located more ventrally ( $Z=32$ rather than $Z=40$ ) and that the right posterior cingulate cluster extended further anteriorly than in the correlational analyses with the TES. The legend in Figure 3 provides $x, y$ and $z$ coordinates for these activations. As shown in Figure 3 at $Z=32$, activation extended into the dorsal aspect of the cingulate at the boundary of its anterior and posterior sections. As expected, the differences were due primarily to activation in relapsers rather than deactivation in the nonrelapsers, although at a lower threshold of $P<0.05$ the nonrelapsers showed deactivation in the left dorsal cingulate (center of mass: $X-6, Y-10, Z 32)$.

\section{DISCUSSION}

This study is the first to show an association between brain activation during drug cue exposure and vulnerability to both treatment dropout and relapse to cocaine use as assessed by the TES (Ling et al, 1997). Those CD patients with worse outcomes (eg relapsers with early dropout, more cocaine use, lower TES) had greater activation of sensory, 


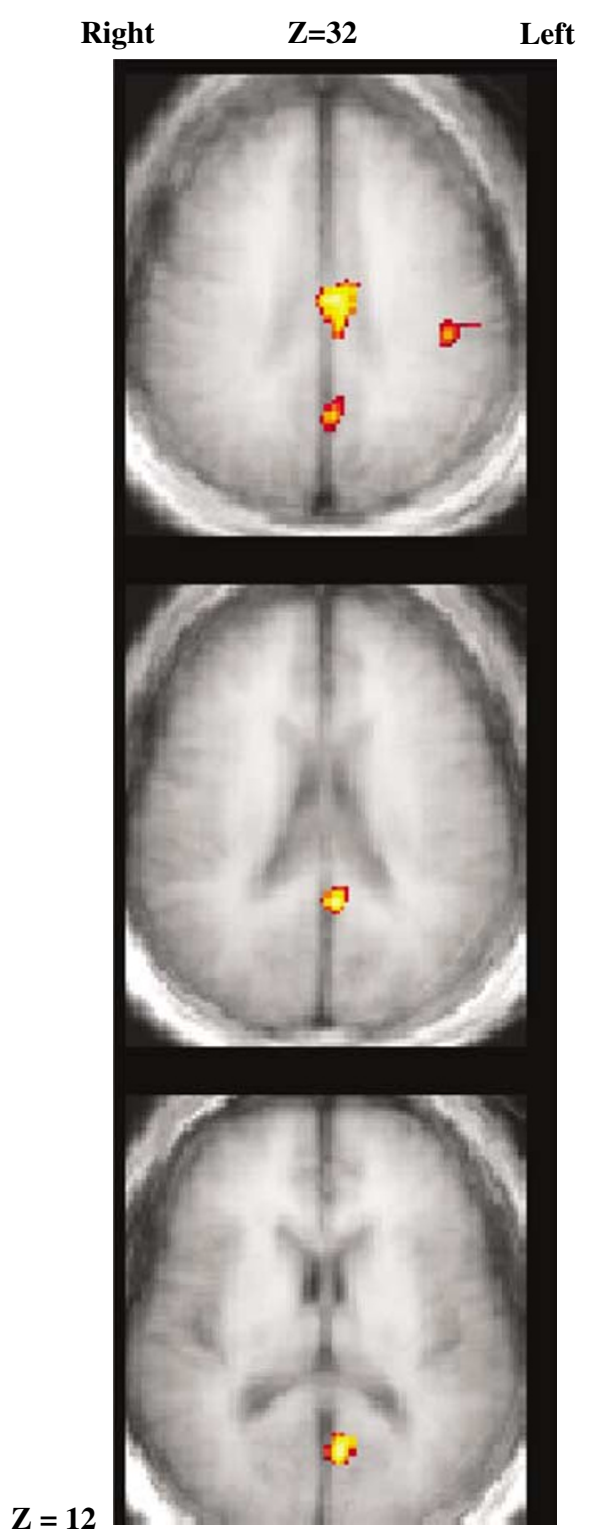

Figure 3 Brain regions showing significant differences in cocaine cueinduced activation (yellow-red) between nonrelapsers and relapsers for the first $30 \mathrm{~s}$ of tape vs baseline. Shown are activations significant at $P<0.0$ I (red) to 0.001 (yellow). Cross-sectional slices are shown from top to bottom at $Z$ levels of 32, 24, and I2. Left $L$ and right $R$ are indicated above the pictures. Slices show posterior cingulate (BA30) in all three crosssections with the following centers of mass: $(X-6, Y-10, Z 32),(X-2$, $Y-50, Z 32),(X-4, Y-60, Z 24),(X-4, Y-65, Z$ I2), and a small area of right precentral BA4 with center of mass at $(X-44, Y-20, Z 32)$.

motor, and limbic cortical areas than $\mathrm{CD}$ patients with better outcomes (eg nonrelapsers with higher TES), particularly during the first $30 \mathrm{~s}$ of cue exposure. When we compared nonrelapsers to relapsers, the association between relapse and posterior cingulate activation was replicated and more extensive than when we considered simple correlations between TES score and brain activation. This greater activation in relapsers than nonrelapsers extended dorsally to the boundary with the anterior cingulate.
In our previous study, three brain areas were significantly more activated in $\mathrm{CD}$ patients than in healthy controls: left precentral (superior), temporal, and anterior cingulate cortices (Wexler et al, 2001). In both the previous and current study, the full $3 \mathrm{~min}$ of tape viewing and image acquisition activated the left precentral and temporal cortices, and in both studies the cingulate was activated before craving was reported (about the first $30 \mathrm{~s}$ of tape viewing). However, the previous and most other studies of cue-induced brain activation differ from the current one in showing activation of the anterior rather than posterior cingulate. Because other studies have used a longer image acquisition time than the current study and have not presented data on early activations before craving is reported, limited comparisons can be made to those studies. The longer acquisition times in previous studies allow engagement of cognitive processes for countering perceived craving and for activation of associated brain areas. The anterior cingulate is one of the brain areas associated with cognitive processing, and the lack of anterior cingulate activation in our current $\mathrm{CD}$ patients even during the full 3 min of acquisition suggests that they made little cognitive effort to counter craving. They may have made less effort due to a shorter period of abstinence before the cue exposure and fMRI scanning for the current $v s$ the previous CD patients (average 5 vs 28 days). The current CD patients may have had less commitment than the previous $C D$ patients to cocaine abstinence, as supported by their drug use during subsequent outpatient care. Half of the current CD patients dropped out of treatment with substantial cocaine use after 2 weeks of abstinence, while the previous $\mathrm{CD}$ patients had been abstinent for a month before imaging and most remained abstinent for several weeks after the imaging. Like CD patients from our previous study, CD patients from studies that showed significant anterior and little posterior cingulate activation have had sustained abstinence when imaged. In other words, more sustained abstinence may allow cognitive processing of cocaine cues and associated anterior cingulate cortex activation, while brief abstinence may not allow this cognitive skill to develop. Instead, strong posterior cingulate activation that occurs below awareness may mark those CD patients who have a high risk of subsequent drug use.

The greater posterior cingulate activation during cue exposure may be related to the greater activation of the sensory association cortex in relapsers (Maddock, 1999). The greater activation of the sensory association cortex in relapsers than nonrelapsers could be interpreted as more active engagement in viewing the cocaine cue tapes. Dorsal cingulate activation is associated with active engagement in viewing arousing images (Taylor et al, 2003). Taylor found dorsal cingulate activation when subjects were asked to rate their affective response to viewing arousing images, but not when they passively viewed the images. Furthermore, posterior cingulate activation is correlated with the magnitude of arousal when patients or healthy subjects are exposed to arousing stimuli (Maddock, 1999). Posterior cingulate activation has been described in previous work on sensory arousal during various types of stimulation including cocaine cue exposure (Garavan et al, 2000).

This posterior cingulate activation below awareness may additionally resemble a type of habit that rapidly associates 
particularly familiar sensory stimuli with heavily reinforced behavior of seeking cocaine (Buccino et al, 2001; Gabriel, 1993; Iacoboni et al, 1999). In animal studies of heavily reinforced habits, such as smoking cocaine was in these subjects, posterior cingulate activity occurs at the initiation of these behaviors (Freeman and Gabriel, 1999; Gabriel, 1993). During the early seconds as well as during the full duration of cue exposure, left precentral motor cortex (BA4) activation was associated with relapse. This BA4 activation may reflect initiation of some motor action associated with seeking cocaine (eg lifting the right arm to smoke cocaine). While this brain activation could not initiate the habit of smoking cocaine during the $\mathrm{AMRI}$ session, the $\mathrm{CD}$ patient in his normal environment is likely to have difficulty inhibiting this first motor component of a habit-like response to the strong sensory stimulation of cocaine cues. Once initiated, this habit could be fully engaged and lead to relapse in the $C D$ patient's normal environment. Thus, while the relapsers watched the videotaped cues, they did not engage the cognitive brain areas associated with these drug cues in other studies, but only appeared to process the incentive value of these cues rapidly and below awareness in the posterior cingulate (Cabeza and Nyberg, 2000; Nyberg et al, 1996). In summary, these neural activations may represent learned drug use habits, as well as processing the incentive valuation and autobiographical memory recall that contribute to motivation for drug use.

The strengths of this study include assessment of brain activation in a drug-free setting and careful outpatient follow-up using three times weekly urine toxicology, but this preliminary study has limitations. First, we had a limited test of the ability of craving to predict relapse in this sample, since the craving response to these cues was only modest, as inpatient studies often report. Second, the drug neutral portion of our video (a CNN reporter giving vegetable prices) could not control for the arousing, emotional, and autobiographical correlates of the drug cue video. However, since all the subjects were exposed to these same videos, any confounding produced by using this type of baseline should have equally affected both treatment outcome groups. Third, the small sample allowed limited adjustments for various sources of variance such as depressive symptoms or extent of drug use. Depressive symptoms were not associated with any specific brain activations, but may have reduced the cue-induced activation of some brain areas such as the anterior cingulate. Depression is associated with anterior cingulate hypo activity and a cue-induced activation could be masked in these CD patients, who overall had elevated scores on the Hamilton Depression scale (Bench et al, 1993; Drevets et al, 1997; George et al, 1997; Mayberg et al, 1997, 1999). However, covariance adjustment for depressive symptoms, as assessed by the Hamilton Depression scale, did not change the locations or strength of the correlations between the TES and brain activation. As to the extent of alcohol or cocaine use, these variables minimally changed correlations between TES outcome and brain activation (eg for posterior cingulate, the correlation dropped from 0.66 to 0.63 ).

In conclusion, the posterior cingulate might function below awareness as a rapid relay for incentive valuation and autobiographical memories to converge with sensory input and then initiate the motor pathways of a learned habit.
This habitual behavior may start during the initial $30 \mathrm{~s}$ of cue exposure before craving has been perceived (Wexler et al, 2001). This rapid cue-induced activation below awareness may make the individual susceptible to cocaine abuse relapse. Confirmatory testing of this neurobiological hypothesis is clearly warranted, but other issues might be simultaneously addressed. For example, does this approach have clinical utility in nondepressed as well as depressed treatment-seeking $C D$ patients? Furthermore, while these brain activations appear to be associated with increased vulnerability to treatment dropout and return to cocaine use, future studies may identify activations that protect from relapse and that can become physiologically defined targets for medication development.

\section{ACKNOWLEDGEMENTS}

This work was supported by the National Institute on Drug Abuse grants P50-DA04060 and DA12762, K05-DA0454 (TRK), K12-DA00366 (MNP), K12-DA00167 (MNP), an NIMH career development award to BEW and by the Veterans Administration, VA New England Mental Illness Research, Education, and Clinical Center.

\section{REFERENCES}

Bench CJ, Friston KJ, Brown RG, Frackowiak RSJ, Dolan RJ (1993). Regional cerebral blood flow in depression measured by positron emission tomography: the relationship with clinical dimensions. Psychol Med 23: 579-590.

Buccino G, Binkofski F, Fink GR, Fadiga L, Fogassi L, Gallese V et al (2001). Action observation activates premotor and parietal areas in a somatotopic manner: an fMRI study. Eur J Neurosci 13: $400-404$

Cabeza R, Nyberg L (2000). Imaging cognition II: an empirical review of 275 PET and fMRI studies. J Cogn Neurosci 12: 1-47.

Childress AR, Mozley PD, McElgin W, Fitzgerald J, Reivich M, O’Brien CP (1999). Limbic activation during cue-induced cocaine craving. Am J Psychiatry 156: 11-18.

Davidson RJ, Irwin W, Anderle MJ, Kalin NH (2003). The neural substrates of affective processing in depressed patients treated with venlafaxine. Am J Psychiatry 160: 64-75.

Drevets WC, Price JL, Simpson Jr JR, Todd RD, Reich T, Vannier $M$ et al (1997). Subgenual prefrontal cortex abnormalities in mood disorders. Nature 386: 824-827.

First MB, Spitzer RL, Gibbon M, Williams JBW (1995). Structured Clinical Interview for DSM-IV. Patient Edition. American Psychiatric Press, Inc.: Washington, DC.

Freeman Jr JH, Gabriel M (1999). Changes of cingulothalamic topographic excitation patterns and avoidance response incubation over time following initial discriminative conditioning in rabbits. Neurobiol Learn Mem 72: 259-272.

Friston KJ, Holmes AP, Worsley KJ, Poline JB, Frith CD, Frackowiak RS (1995). Statistical parametric maps in functional imaging: a general linear approach. Hum Brain Mapp 4: 189-210.

Gabriel M (1993). Discriminative avoidance learning: a model system. In: Vogt BA, Gabriel M (eds). Neurobiology of Cingulate Cortex and Limbic Thalamus. Birkhauser: Boston, MA. pp 478-523..

Garavan H, Pankiewicz J, Bloom A, Cho JK, Sperry L, Ross TJ et al (2000). Cue-induced cocaine craving: neuroanatomical specificity for drug users and drug stimuli. Am J Psychiatry 157: 1789-1798.

George MS, Ketter TA, Parekh PI, Rosinsky N, Ring HA, Pazzaglia PJ et al (1997). Blunted left cingulate activation in mood disorder 
subjects during a response interference task (the Stroop). J Neuropsychiatry Clin Neurosci 9: 55-63.

Hamilton MA (1960). A rating scale for depression. J Neurol Neurosurg Psychiatry 23: 56-62.

Hays WL (1988). Statistics. Holt, Rinehart \& Winston: Orlando, FL. Iacoboni M, Woods RP, Brass M, Bekkering $\mathrm{H}$, Mazziotta JC, Rizzolatti G (1999). Cortical mechanisms of human imitation. Science 286: 2526-2528.

Ling W, Shoptaw S, Wesson D, Rawson RA, Compton M, Klett CJ (1997). Treatment effectiveness score as an outcome measure in clinical trials. NIDA Res Monogr 175: 208-220.

Maas LC, Lukas SE, Kaufman MJ, Weiss RD, Daniels SL, Rogers VW et al (1998). Functional magnetic resonance imaging of human brain activation during cue-induced cocaine craving. Am J Psychiatry 155: 124-126.

Maddock RJ (1999). The retrosplenial cortex and emotion: new insights from functional neuroimaging of the human brain. Trends Neurosci 22: 310-316.

Manly B (1997). Randomization, Bootstrap and Monte Carlo Methods in Biology. Chapman \& Hall: London, UK.

Mayberg HS, Brannan SK, Mahurin RK, Jerabek PA, Brickman JS, Tekell JL et al (1997). Cingulate function in depression: a potential predictor of treatment response. Neuroreport 8: 1057-1061.

Mayberg HS, Liotti M, Brannan SK, McGinnis S, Mahurin RK, Jerabek PA et al (1999). Reciprocal limbic-cortical function and negative mood: converging PET findings in depression and normal sadness. Am J Psychiatry 156: 675-682.

Mucha RF, Geier A, Stuhlinger M, Mundle G (2000). Appetitive effects of drug cues modelled by pictures of the intake ritual: generality of cue-modulated startle examined with inpatient alcoholics. Psychopharmacology (Berlin) 151: 428-432.

Nyberg L, Cabeza R, Tulving E (1996). PET studies of encoding and retrieval: the HERA model. Psychon Bull Rev 3: 135-148.

Shaywitz SE, Shaywitz BA, Pugh KR, Fulbright RK, Skudlarski P, Mencl WE et al (1999). Effect of estrogen on brain activation patterns in postmenopausal women during working memory tasks. J Am Med Assoc 281: 1197-1202.

Skudlarski P, Gore JC (1999). Randomization in multi-subject fMRI studies: ROC analyses (abstract). Neuroimage 9: 591.

Talairach J, Tournoux P (1988). Co-Planar Stereotaxic Atlas of the Human Brain. Thieme Medical: New York.

Taylor SF, Phan KL, Decker LR, Liberzon I (2003). Subjective rating of emotionally salient stimuli modulates neural activity. Neuroimage 18: 650-659.

Tiffany ST, Carter BL (1998). Is craving the source of compulsive drug use? J Psychopharmacol (Oxford) 12: 23-30.

Wexler BE, Gottschalk CH, Fulbright RK, Prohovnik I, Lacadie CM, Rounsaville BJ et al (2001). Functional magnetic resonance imaging of cocaine craving. Am J Psychiatry 158: 86-95.

Wilson SJ, Sayette MA, Fiez JA (2004). Prefrontal responses to drug cues: a neurocognitive analysis. Nat Neurosci 7: 211-214. 